\title{
Thermoelectric properties of oil fly ash-derived carbon nanotubes coated with polypyrrole
}

\author{
Cite as: J. Appl. Phys. 128, 235104 (2020); doi: 10.1063/5.0031438 \\ Submitted: 30 September 2020 - Accepted: 24 November 2020 . \\ Published Online: 16 December 2020

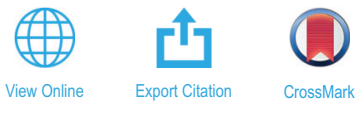 \\ Numan Salah, , ,a) (iD Nuha A. Alhebshi, ${ }^{2}$ (D) Yousef N. Salah, ${ }^{3}$ Husam N. Alshareef, ${ }^{4}$ (D) and Kunihito Koumoto ${ }^{1,5}$ (iD

\begin{abstract}
AFFILIATIONS
${ }^{7}$ Center of Nanotechnology, King Abdulaziz University, Jeddah 21589, Saudi Arabia

${ }^{2}$ Physics Department, Faculty of Science, King Abdulaziz University, Jeddah 21589, Saudi Arabia

${ }^{4}$ Materials Science and Engineering, Physical Science and Engineering Division, King Abdullah University of Science and

Technology (KAUST), Thuwal 23955-6900, Saudi Arabia

${ }^{5}$ Nagoya Industrial Science Research Institute, Nagoya 464-0819, Japan
\end{abstract} \\ ${ }^{3}$ Department of Chemical and Materials Engineering, Faculty of Engineering, King Abdulaziz University, Rabigh 21911, Saudi Arabia
}

Note: This paper is part of the Special Topic on Physics and Applications of Nanotubes.

${ }^{a)}$ Author to whom correspondence should be addressed: nsalah@kau.edu.sa and alnumany@yahoo.com

\begin{abstract}
Oil fly ash has been reported to be suitable for producing low-cost carbon nanotubes (CNTs). These CNTs exhibit zigzag curved walls with an almost bamboo-like structure. Owing to this structure, these CNTs exhibit very low thermal conductivity as compared to other graphitic carbon materials. They also exhibit relatively low electrical conductivity. However, they exhibit a Seebeck coefficient comparable to that of commercially available CNTs. Therefore, it is of great importance to evaluate the thermoelectric (TE) properties of oil fly ash-derived CNTs. In this study, the TE properties of oil fly ash-derived CNTs were investigated. The CNTs were further coated with polypyrrole (PPy) to enhance their TE performance. PPy was used for the modification because of its attractive TE properties and its suitability as a binder for CNTs. The PPy coating significantly enhanced the electrical conductivity of the CNTs from $\sim 500$ to $\sim 1300 \mathrm{~S} / \mathrm{m}$ at room temperature. A small increase in the Seebeck coefficient was also observed. The power factor value increased from 0.1 to $0.6 \mu \mathrm{W} / \mathrm{m} \mathrm{K}^{2}$. At $440 \mathrm{~K}$, the power factor value was $1.4 \mu \mathrm{W} / \mathrm{m} \mathrm{K}^{2}$. The thermal conductivity of the CNTs $(\sim 1 \mathrm{~W} / \mathrm{m} \mathrm{K})$ decreased significantly by a factor of 10 after the modification with PPy. The power generation characteristics of a single leg module made up of the p-type coated CNTs were investigated under real-time conditions in air. The results demonstrated the potential of the oil fly ash-derived CNTs coated with PPy for applications as TE materials.
\end{abstract}

Published under license by AIP Publishing. https://doi.org/10.1063/5.0031438

\section{INTRODUCTION}

Exploiting renewable energy resources such as solar power, wind power, hydropower, biomass, geothermal, and marine energy, which are sustainable, is imperative to meet future energy demands. The heat generated by the Sun on the Earth is unlimited and can be used as an abundant renewable energy source, particularly in the Sun Belt. The waste heat emitted by combustion engines and machines is another source that might be utilized for generating energy using proper devices and systems. Thermoelectric (TE) devices are tools designed using semiconducting materials that can convert heat into electricity. ${ }^{1}$ Several materials and structures ${ }^{2-5}$ including nanostructures $^{6,7}$ have been investigated as TE materials. However, developing highly efficient TE materials is still a challenge. It is of great importance to develop materials/composites with excellent TE properties such as high efficiency (high electrical conductivity, high Seebeck coefficient, low thermal conductivity), good thermal stability, low cost, scalability, nontoxicity, and environmental friendliness.

Carbon nanomaterials, particularly carbon nanotubes (CNTs), have gained significant attention owing to their unique mechanical and electrical properties. ${ }^{8}$ CNTs have been extensively investigated for various applications, including TE materials. ${ }^{9-12}$ CNTs are basically tube-shaped hexagonal rings of carbon atoms with diameters in the nanometer scale. Various methods have been reported for preparing CNTs. ${ }^{8,13-15}$ However, these methods offer low yield and 
are expensive. CNTs exhibit attractive properties for TE applications, especially high electrical conductivity. ${ }^{16}$ However, the thermal conductivity of CNTs is considerably high, ${ }^{17}$ which suppresses their TE properties by reducing the figure of merit $(z T)$. The latter is a dimensionless parameter used to evaluate the performance of TE materials and is given by $S^{2} \sigma \mathrm{T} / \kappa$, where $S$ is the Seebeck coefficient, $\sigma$ is the electrical conductivity, $T$ is the absolute temperature, and $\kappa$ is the thermal conductivity.

Recently, Salah et al. ${ }^{18,19}$ prepared CNTs using ultrasonicated oil fly ash as the precursor and catalyst. They successfully used these CNTs for some applications. ${ }^{20-22}$ These CNTs are multiwalled and showed considerable mechanical reinforcements for different polymers. ${ }^{22}$ Moreover, they were found to be superior as lubricant additives compared with other commercial carbon nanomaterials. ${ }^{20,21}$ This indicates that the CNTs of oil fly ash have excellent mechanical properties with good stability. Furthermore, these CNTs exhibited zigzag curved walls with an almost bamboo-like structure. Owing to this structure, the CNTs exhibited very low thermal conductivity as compared to CNTs derived from common graphitic carbon materials. Their electrical conductivity was also relatively low. However, these CNTs exhibited a Seebeck coefficient comparable to that of commercially available CNTs. Therefore, it is of great importance to evaluate the TE properties of oil fly ashderived CNTs. The low cost of these CNTs and their scalability is another attractive advantage to utilize them as TE materials. They have the potential to be designed as large TE plates for houses (window frames, walls, etc.) in hot countries. TE plates made up of these CNTs can act as both heat insulators and TE power generators because of the low thermal conductivity of the CNTs.

TE devices are composed of films or legs/plates made up of bulk or nanostructured materials. Some of the promising TE materials exist in the powder form, which require proper binders. In the absence of proper binders, cracks might be formed during the operation process, which might affect the performance of the TE devices. Such cracks reduce both the effective electrical and thermal conductivities of TE devices. ${ }^{23}$ To address this issue, a proper organic binder should be employed. In this regard, polypyrrole (PPy) is considered the most suitable binder owing to its excellent TE properties. ${ }^{24,25} \mathrm{PPy}$ has been incorporated into commercial carbon nanomaterials to produce high-performance TE composites. ${ }^{9-11,26}$ In this study, CNTs were prepared using oil fly ash and their TE properties were investigated. These CNTs were further coated with PPy to improve their TE performance. PPy was used for the modification of the CNTs owing to its attractive TE properties and suitability as a CNT binder. The PPy coating significantly improved the electrical conductivity of the CNTs and reduced their thermal conductivity. The CNTs were characterized using various analytical techniques. To investigate the TE performance of the CNTs, the power generation characteristics of singleleg modules made of CNTs, PPy, and CNTs/PPy were investigated under real-time conditions, in air.

\section{EXPERIMENTAL DETAILS}

\section{A. Material synthesis}

The CNTs were prepared according to the method reported previously by Salah et al. ${ }^{18,19}$ The PPy-coated CNTs were prepared
TABLE I. Raw materials used for coating the CNTs with PPy at different layer thicknesses.

\begin{tabular}{lcccc}
\hline \hline Sample & $\begin{array}{c}\text { CNTs } \\
(\mathrm{mg})\end{array}$ & $\begin{array}{c}\text { SDs } \\
(\mathrm{mg})\end{array}$ & $\begin{array}{c}\mathrm{Py}(\mathrm{ml}) \\
(\text { in } 200 \mathrm{ml} \mathrm{DI})\end{array}$ & $\begin{array}{c}\mathrm{FeCl}_{3}(\mathrm{mg}) \\
(\mathrm{in} 200 \mathrm{ml} \mathrm{DI})\end{array}$ \\
\hline CNT-PPy 0.05 & 600 & 37.5 & 0.05 & 117 \\
CNT-PPy 0.1 & & 75 & 0.1 & 234 \\
CNT-PPy 0.2 & & 150 & 0.2 & 468 \\
CNT-PPy 0.3 & & 225 & 0.3 & 702 \\
CNT-PPy 0.4 0.4 & 300 & 0.4 & 936 \\
\hline \hline
\end{tabular}

using sodium dodecyl sulfate (SDS) as the surfactant and a small amount of Pyrrole (Py) along with an appropriate amount of $\mathrm{FeCl}_{3}$ as the initiator. The reagents used were purchased from Sigma-Aldrich and were of analytical grade (99.99\%). Pure PPy was prepared by dissolving $300 \mathrm{mg}$ of SDS in $200 \mathrm{ml}$ of DI water. To the resulting solution, $0.4 \mathrm{ml}$ of Py was added under continuous stirring for $30 \mathrm{~min}$. An appropriate amount of $\mathrm{FeCl}_{3}(936 \mathrm{mg})$ was separately dissolved in $200 \mathrm{ml}$ of DI water. This solution was then added dropwise to the Py solution. The resulting dispersion was magnetically stirred for another $24 \mathrm{~h}$ and was then filtered and washed several times with distilled water and ethanol to remove the surfactant and unreacted species. The resulting powder was dried at $60{ }^{\circ} \mathrm{C}$. The polymerization of PPy on the surface of the CNTs was carried out by sonicating the desired amount of CNTs $(600 \mathrm{mg})$ in $200 \mathrm{ml}$ of DI water for $1 \mathrm{~h}$ and then adding the desired amounts of SDS and pyrrole to this solution under continuous stirring for 30 min. To this solution, a solution of $\mathrm{FeCl}_{3}$ in $200 \mathrm{ml}$ of DI water was added dropwise and the resulting mixture was then stirred and dried under the same conditions as those used in the case of pure PPy. The amounts of the CNTs and other materials used for coating are listed in Table I.

\section{B. Characterization and TE measurements}

The morphologies of the pure and PPy-coated CNTs were examined using scanning electron microscopy (SEM) (JSM-7500F, JEOL, Japan) and transmission electron microscopy (TEM) (JEM 2100F, JEOL). The Raman spectra of the samples were recorded on a micro-Raman spectroscope (Thermo Fisher Scientific, USA). Attenuated total reflection Fourier-transform infrared (ATR-FTIR) spectroscopy (Thermo Fisher Scientific) was used to obtain the FTIR spectra of the samples. The x-ray photoelectron spectroscopy (XPS) measurements of the samples were carried out on a PHI 5000 VersaProbe, Japan. The pellets of the pure and PPy-coated CNTs were prepared using a manual hydraulic press. The diameter of the pellets was $13 \mathrm{~mm}$ and the thickness was varied from 1 to $2 \mathrm{~mm}$. The pellets were annealed in a vacuum furnace at $440 \mathrm{~K}$ for $60 \mathrm{~min}$.

To investigate the electrical and thermal properties of the pellets, their resistivities and Seebeck coefficients were measured. An LSR-3 Linseis-Seebeck coefficient and electric resistivity system (Linseis, Germany) was used in a helium atmosphere for measuring the resistivities and Seebeck coefficients of the pellets. The heating rate and temperature gradient between the hot and cold sides were 
fixed at $5{ }^{\circ} \mathrm{C} / \mathrm{min}$ and $50^{\circ} \mathrm{C}$, respectively. A laser flash thermal conductivity analyzer (LFA-1000, Linseis) was used to measure the thermal conductivity of the samples. The measurements were carried out perpendicular to the surface of the pellet. A manual hydraulic press was used to manufacture the rectangular $\left(2 \times 6 \times 10 \mathrm{~mm}^{3}\right)$ single-leg modules of pure CNTs, PPy, and the PPy-coated CNTs. These modules were fixed on an alumina substrate. Aluminum electrodes were used to attach both sides to the measurement system. To test the modules, a hot plate was heated to the maximum temperature $(440 \mathrm{~K})$. A high sensitivity $I-V$ measurement system (Keithley Instruments, USA) was used to measure the output voltage and current.

\section{RESULTS AND DISCUSSION}

The morphologies and microstructures of the CNTs were examined using SEM and TEM (Fig. 1). ${ }^{18,19}$ As can be observed from the SEM images shown in Figs. 1(a) and 1(b), the CNTs exhibited almost uniform dimensions. The CNTs had a diameter of 25-40 nm and a length of a few micrometers. Moreover, these

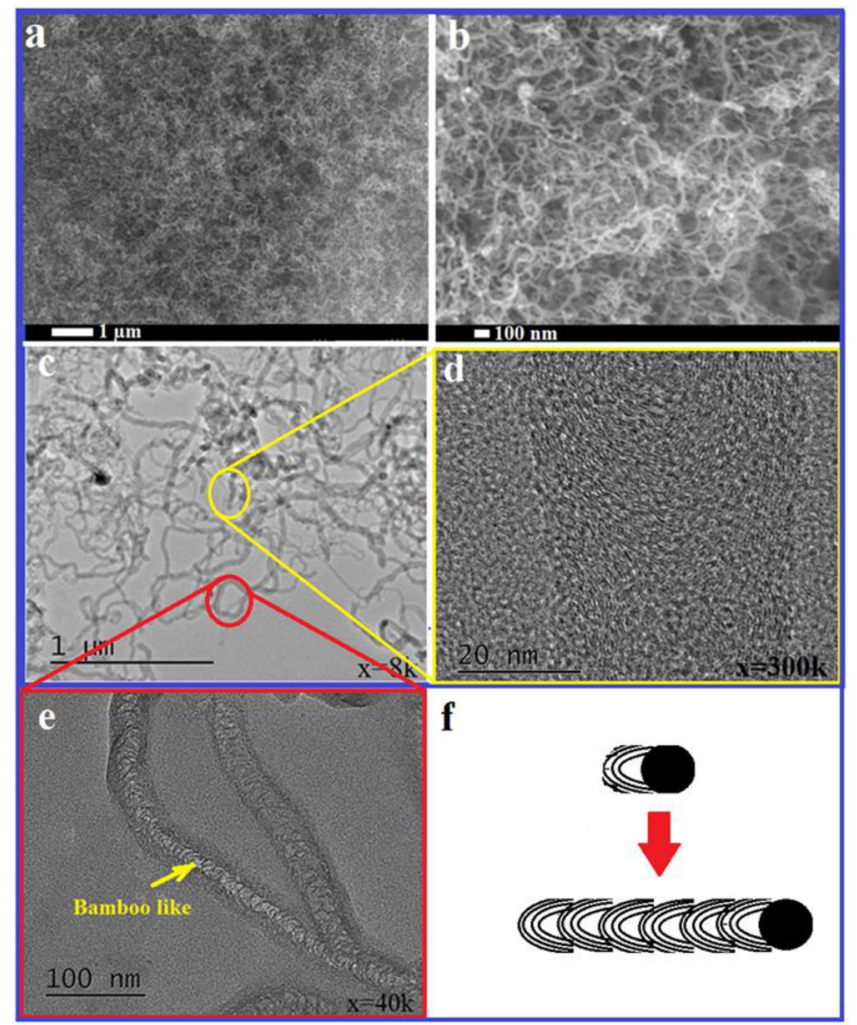

FIG. 1. SEM [(a) and (b)] and TEM [(c)-(e)] images of the CNTs. (d) HRTEM image showing the zigzagged multiwalls of the CNTs. The image in (e) shows the bamboo-like structure of the CNTs. This structure is illustrated in (f). The curved walls started growing around the catalyst particles (the black dot). The CNTs were grown by the CVD method on a treated oil fly ash containing Fe and $\mathrm{Ni}$, which acted as catalysts.
CNTs showed a zigzag structure, which was significantly different from those of commercially available CNTs. The TEM and highresolution TEM (HRTEM) images of the as-grown CNTs are shown in Figs. 1(c)-1(e). As can be observed, the zigzag multiwall CNTs exhibited dimensions close to those observed by the SEM images. These CNTs were grown by the chemical vapor deposition method on treated oil fly ash containing small concentrations of $\mathrm{Fe}$ and $\mathrm{Ni}$, which acted as catalysts. The CNTs showed a bamboo-like structure [Fig. 1(e)]. This structure is illustrated in Fig. 1(f). We believe that during the growth of these CNTs, curved multiwalls originated around the existing catalyst particles and continued their growth, resulting in the formation of bamboo-like structures.

The morphology and microstructure of PPy were also examined using SEM and TEM (Fig. 2). As can be observed from the SEM images (a) and (b), the PPy showed a continuous sheet-like structure composed of spherical nanoparticles. These nanoparticles were strongly connected with each other and showed good particle size uniformity $(50-100 \mathrm{~nm})$. Figures 2 (c) and 2(d) show the TEM and HRTEM images of PPy. These images showed nanoparticles with sizes and shapes similar to those of the nanoparticles observed by SEM. The HRTEM image of PPy showed the presence of a short lattice arrangement in the zigzagged patterns. This atomic resolution image also showed some pentagonal and hexagonal rings. These results are consistent with those reported previously. ${ }^{27}$

Figure 3 shows the SEM images of the uncoated and PPy-coated CNTs with different PPy layer thicknesses. It can be clearly observed from the images that the PPy coating significantly affected the diameter of the CNTs. A systematic coating could be

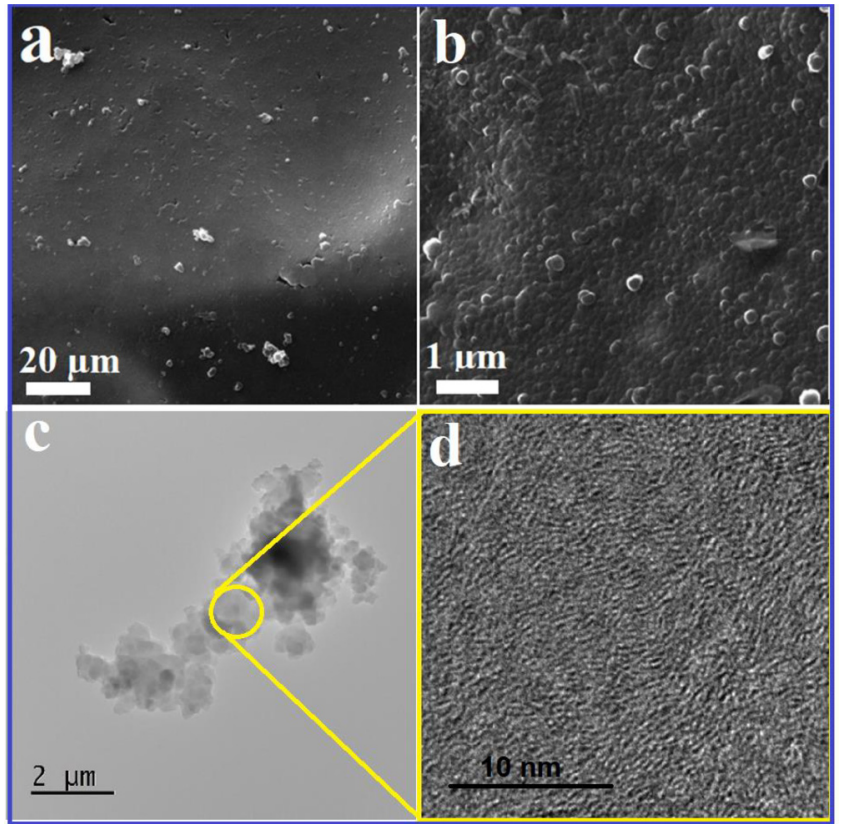

FIG. 2. SEM [(a) and (b)] and TEM [(c) and (d)] images of pure PPy. (d) shows the HRTEM image of PPy, revealing its short-range graphitic structure. 

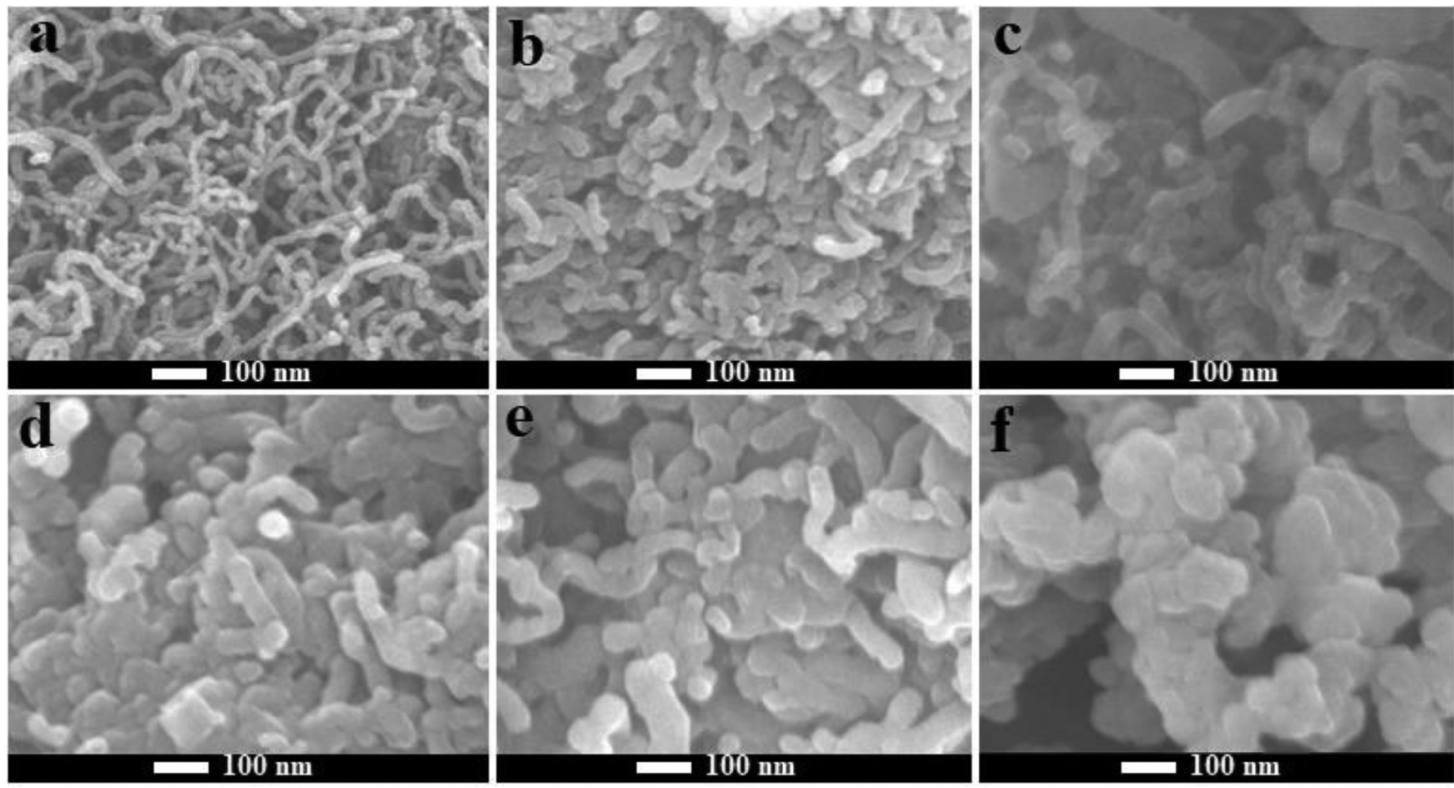

FIG. 3. SEM images of the CNTs without (a) and with PPy coating with different layer thicknesses [(b): $0.05,(c): 0.1,(d): 0.2,(e): 0.3,(f): 0.4 \mathrm{ml}]$.

observed. The diameter of the coated CNTs increased with an increase in the thickness of the coated layer (increasing the amount of PPy from 0.05 to $0.4 \mathrm{ml}$ ). The CNTs coated with $0.4 \mathrm{ml} \mathrm{PPy}$ [Fig. 3(f)] were distorted and showed nanoparticle agglomeration on the surface. The CNTs coated with smaller amounts of PPy retained their tubular structure. However, the morphology of the CNTs coated systematically with PPy in this study was better than that of PPy-coated CNT@MnO 2 by PPy. ${ }^{28}$

The microstructures of the uncoated CNTs and PPy-coated CNTs with different PPy layer thicknesses (increasing the amount of PPy from 0.05 to $0.4 \mathrm{ml}$ ) were also examined using TEM (Fig. 4). The diameter of the coated CNTs increased systematically with an increase in the amount of PPy from 0.05 to $0.4 \mathrm{ml}$ [Figs. 4(b)-4(f)]. The HRTEM images of the CNTs without and (h) with PPy coating were also recorded. The uncoated CNTs showed multiwalls similar to those observed in Fig. 1(d). On the other hand, the CNTs coated with PPy were thicker and did not exhibit clear multiwalls. The PPy layer could only be seen in the CNTs coated with $0.05 \mathrm{ml}$ of PPy. However, higher amounts of PPy could create thicker layers of PPy. The densely distributed pentagonal and hexagonal rings in this thick layer covered the CNTs. A single PPy nanoparticle could also be observed in Fig. 4(h), which showed a microstructure similar to that shown in Fig. 2(d).

The CNTs were also analyzed using Raman spectroscopy, FTIR spectroscopy, and XPS, and the results are shown in Figs 5, 6, and 7, respectively. The Raman spectra of the CNTs with different PPy layer thicknesses are shown in Fig. 5. The spectra of the uncoated CNTs and pure PPy are also shown. The uncoated CNTs showed two prominent intense bands at 1340 and $1595 \mathrm{~cm}^{-1}$ corresponding to the in-plane vibration of graphite ( $\mathrm{G}$ band) and the disorder-induced vibration (D-band), respectively. ${ }^{19}$ The pure PPy showed two strong bands at around 1360 and $1570 \mathrm{~cm}^{-1}$ corresponding to the $\mathrm{C}-\mathrm{C}$ and $\mathrm{C}=\mathrm{C}$ in-ring vibration modes of the polymer backbone, respectively. ${ }^{29,30}$ The pure PPy also showed weak bands at around 950 and $1080 \mathrm{~cm}^{-1}$. The first one can be ascribed to the ring deformation associated with the di-cation (di-polaron), while the second one corresponds to the $\mathrm{C}-\mathrm{H}$ in-plane deformation. ${ }^{31}$ The spectra of the PPy-coated CNTs are similar to those of the pure PPy, but with a systematic decrease in the band intensities by increasing coating thickness. Additionally, a shift in the $1570 \mathrm{~cm}^{-1}$ band of PPy to the larger wavenumber is observed in the coated samples. This decrease and band shift might indicate a significantly strong coupling and charge transfer between PPy and CNTs. An efficient $\pi-\pi$ stacking between PPy chains and CNTs particularly for the PPy of higher layer thickness might have resulted in a significant reduction in the band intensities. However, the oil fly ash-derived CNTs prepared in this study might be further functionalized with proper functional groups such as carboxyl groups simply by using nitric acid $\left(\mathrm{HNO}_{3}\right)^{31}$ or sulfuric acid $\left(\mathrm{H}_{2} \mathrm{SO}_{4}\right)$ to improve the composite formation/interaction.

The FTIR spectra of the coated CNTs with different PPy layer thicknesses are shown in Fig. 6. The spectra of pure CNTs and PPy are also shown. The CNTs showed major bands at 1540 and $1640 \mathrm{~cm}^{-1}$. The first one can be ascribed to the carboxylate/carboxylic acid groups present in the CNTs, ${ }^{22}$ while the second band corresponds to the $\mathrm{C}=\mathrm{C}$ stretching of the CNTs. Pure PPy showed peaks at around $860,1031,1166,1458$, and $1550 \mathrm{~cm}^{-1}$. The peak at $860 \mathrm{~cm}^{-1}$ can be attributed to $\mathrm{C}-\mathrm{H}$ wagging, while that at $1040 \mathrm{~cm}^{-1}$ corresponds to the $\mathrm{N}-\mathrm{H}$ stretching vibration and $\mathrm{C}-\mathrm{H}$ 

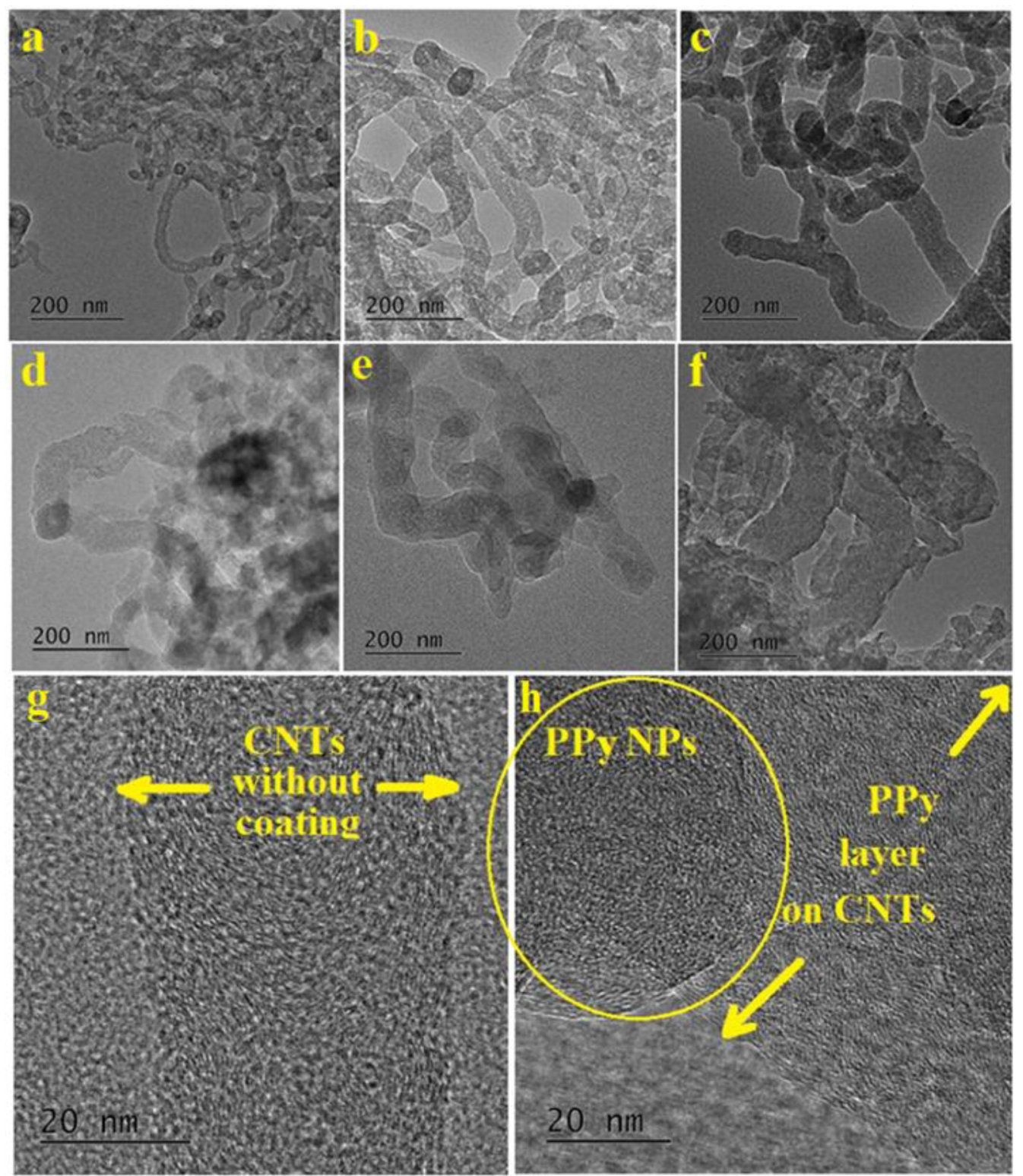

FIG. 4. TEM images of the CNTs without (a) and with PPy coating at different layer thicknesses [(b): 0.05 , (c): 0.1 , (d): 0.2 , (e): 0.3 , (f): $0.4 \mathrm{ml}$. HRTEM images of the CNTs without and (h) with PPy coating (g). Atomic resolution for a single PPy NP can also be seen in (h).

in-plane deformation. ${ }^{32,33}$ The two bands located at 1170 and $1450 \mathrm{~cm}^{-1}$ can be attributed to the $\mathrm{C}-\mathrm{N}$ stretching vibration mode in the PPy ring, while that at $1550 \mathrm{~cm}^{-1}$ can be ascribed to the C-C symmetric ring vibration. ${ }^{33}$ The FTIR spectra of the CNTs coated with PPy were similar to those of the pure CNTs and PPy. This suggests that all the characteristic peaks of PPy and the CNTs were present in the coated nanotubes. The intensity of the $\mathrm{C}-\mathrm{H}$ peak decreased with an increase in the coating thickness. This might be attributed to the slight deformation of the PPy backbone.
It is also possible that this peak decreased due to the $\pi-\pi$ stacking between the PPy chains and CNTs. The other peaks did not show significant changes in their positions or intensities.

The XPS survey profiles of the pure CNTs, PPy, and CNTs with different PPy layer thicknesses are shown in Fig. 7. The C1s and O1s OKLL peaks could be clearly observed in the spectra of the pure samples (e.g., CNTs and PPy). Additional small N1s and S2s peaks were observed in the spectrum of $\mathrm{PPy}$. The $\mathrm{C} 1 \mathrm{~s} / \mathrm{O} 1 \mathrm{~s}$ ratio of the pure CNTs was higher than that of PPy. The XPS profiles of 


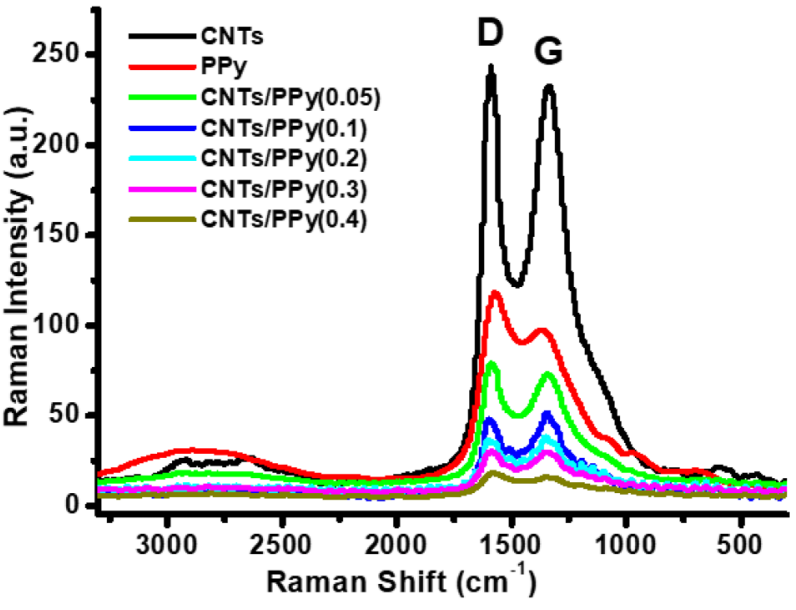

FIG. 5. Raman spectra of pure CNTs, pure PPy, and the PPy-coated CNTs with different PPy layer thicknesses.

the pure CNTs and PPy obtained in this study were similar to those reported in the literature for PPy nanoparticles and nanorods ${ }^{30}$ and CNTs. ${ }^{34}$ In the case of the PPy-coated CNTs, the intensity of the O1s band decreased slightly with an increase in the coating thickness. A weak $\mathrm{Cl} 2 \mathrm{p}$ band was observed at around $200 \mathrm{eV}$ in the coated CNTs, particularly at higher PPy thicknesses (e.g., 0.3 and $0.4 \mathrm{ml}$ ). This peak might be attributed to the $\mathrm{Cl}$ introduced by the use of $\mathrm{FeCl}_{3}$ as the initiator. The concentration of the latter was high during the CNT coating by 0.3 and $0.4 \mathrm{ml}$ of PPy; therefore, a residue of $\mathrm{Cl}$ is most probably formed by this band.

Figures $8(\mathrm{a})-8(\mathrm{~g})$ show the C1s narrow scan profiles of PPy and the uncoated and coated CNTs. The C1s band of the CNTs

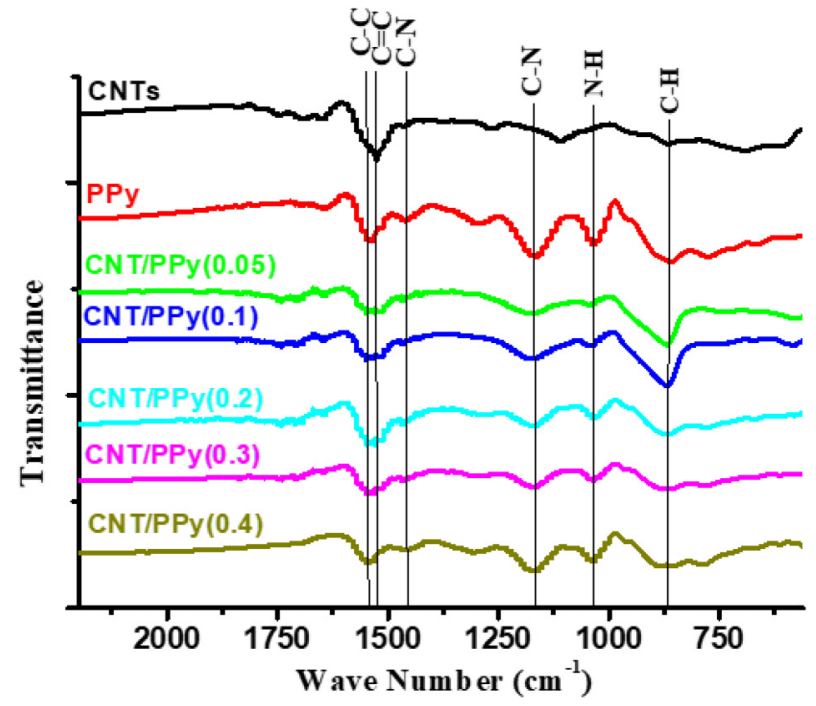

FIG. 6. FT-IR spectra of PPy and the CNTs without and with PPy.

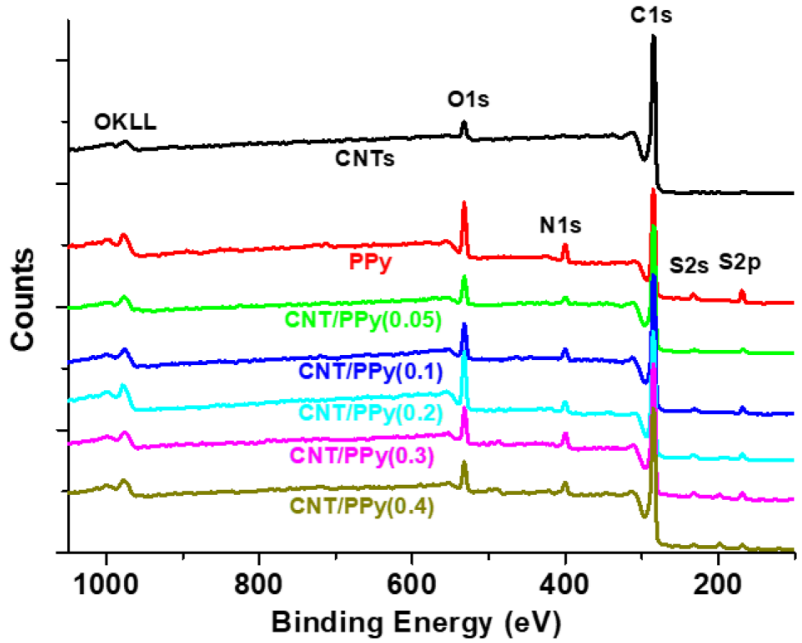

FIG. 7. XPS survey profiles of PPy and the CNTs with and without PPy coating.

could be deconvoluted into three components at 284.77, 286.50, and $289.34 \mathrm{eV}$ corresponding to the $\mathrm{C}=\mathrm{C}, \mathrm{C}-\mathrm{O}$, and $\mathrm{COOH}$ groups, respectively [as Fig. 8(a)]. The major band was located at $284.77 \mathrm{eV}$, which occupied an area of more than $80 \%$. This band corresponded to the $\mathrm{sp}^{2}$ carbon of the CNTs. ${ }^{19}$ The weak band located at $289.34 \mathrm{eV}$ corresponded to the formation of a functional group on the surface of the CNTs. PPy showed C1s bands at 284.83, 286.28 , and $289.05 \mathrm{eV}$ corresponding to the $\mathrm{C}=\mathrm{C}$ (corresponding to $\alpha$ carbon atoms in the Py ring), $\mathrm{C}-\mathrm{N}^{+}$(bonds between carbon and nitrogen in the PPy structure), and $\mathrm{C}=\mathrm{O}$ (assigned to $\mathrm{C}=\mathrm{O}$ species), ${ }^{30}$ respectively [Fig. $8(\mathrm{~b})$ ]. The latter can also be ascribed to the carboxylic acid $(\mathrm{COOH})$ group. ${ }^{11,35}$ The $\mathrm{C}=\mathrm{C}$ band was the most intense in the spectrum of pure PPy; however, its intensity was lower than that of the $\mathrm{C}=\mathrm{C}$ band in the CNTs. The spectra of the coated CNTs with different PPy layer thicknesses [Figs. 8(c)-8(g)] were almost similar to the spectra of the uncoated CNTs and pure PPy; however, their band intensities were intermediate to those of the pure CNTs and PPy (lower than those of the pure CNTs but higher than those of PPy). No significant changes were observed in the peak positions. This indicates that a perfect PPy coating was formed on the surface of the CNTs with no extra bonds or crosslinking in the contact surfaces. The excellent $\pi-\pi$ stacking between PPy chains and CNTs might also assist in stabilizing the binding energies of the elements involved in both PPy and CNTs.

The TE properties of the pure CNTs and PPy are shown in Figs. 9(a)-9(c). The measured electrical conductivity as a function of temperature for both the materials was found to be approximately $500 \mathrm{~S} / \mathrm{m}$ at room temperature (RT). This value increased with an increase in temperature up to $440 \mathrm{~K}$ reaching $700 \mathrm{~S} / \mathrm{m}$ for the CNTs and $1200 \mathrm{~S} / \mathrm{m}$ for PPy. The relatively low electrical conductivity of the oil fly ash-derived CNTs prepared in this study as compared to those of the CNTs reported in the literature ${ }^{36}$ might be attributed to their unique microstructure, as shown in Fig. 1. The bamboo-like CNTs and their zigzagged walls hindered the 

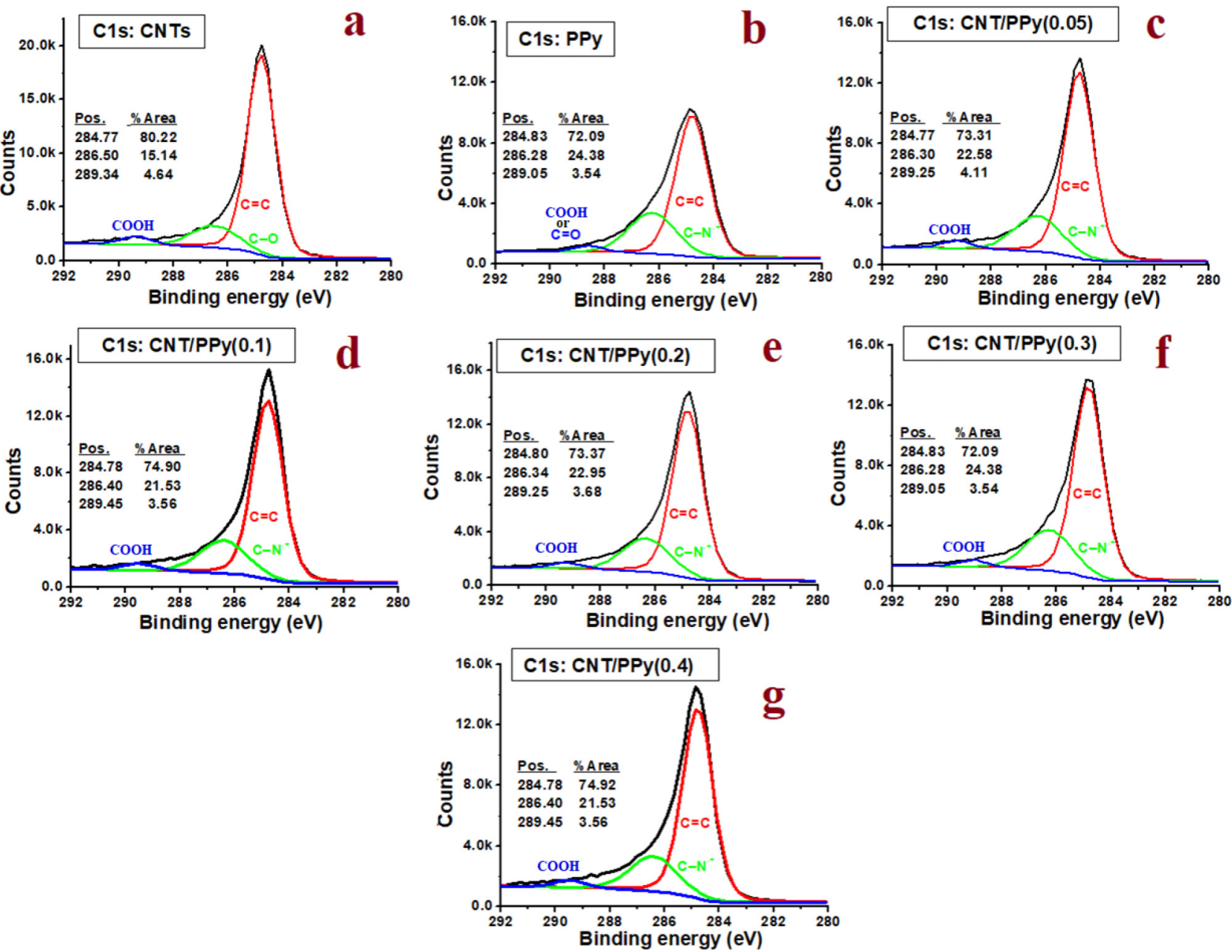

FIG. 8. C1s XPS profiles of PPy and the CNTs with and without PPy coating.

mobility of charge carriers, thus reducing the electrical conductivity. There might be another reason for this low electrical conductivity, which is the low carrier concentration in the CNTs of oil fly ash. It is understood that oxygen-containing graphitic carbon materials are p-type semiconducting. ${ }^{10}$ The present CNTs have a limited amount of oxygen as shown in Fig. 7(a) and also as reported previously from the XPS analysis; ${ }^{19}$ therefore, it might not be enough to enrich the CNTs with carrier holes and thus showed only low electrical conductivity. The electrical conductivity of the PPy was comparable to or even higher than those with similar structures or morphologies as reported in the literature., ${ }^{9,26}$

Figure 9(b) shows the Seebeck coefficient of the CNTs and $\mathrm{PPy}$ as a function of temperature over the temperature range of 300-440 K. The RT Seebeck coefficients of the CNTs and PPy were 15 and $21 \mu \mathrm{V} / \mathrm{K}$, respectively. With an increase in temperature to $440 \mathrm{~K}$, the Seebeck coefficient of the CNTs increased slowly to approximately $20 \mu \mathrm{V} / \mathrm{K}$, while that of the PPy remained almost unchanged. It has been reported that the Seebeck coefficient CNTs at RT can vary over a wide range $(\sim 13-70 \mu \mathrm{V} / \mathrm{K})$, depending mainly on the dopant type and its concentration. P-type CNTs are produced by including oxidative dopants such as oxygen, acids, or chemical oxidants. ${ }^{10}$ Figures 7 (a) and 7(b) clearly show that a considerable number of oxygen compounds/groups were present in the grown CNTs. Therefore, the CNTs prepared in this study were p-type semiconductors. The electrical conductivity of the CNTs increased with an increase in temperature, which is a common trend for semiconductors. The Seebeck coefficient of the PPy prepared in this study was considerably higher than those of similar structures and morphologies reported in the literature. ${ }^{11,26}$ This might be due to the morphology of PPy, which showed a continuous sheet composed of tightly connected spherical nanoparticles [Figs. 2(a) and 2(b)]. At the interfaces of the nanoparticles, potential barriers might be formed, which allow charge transport only for high-energy carriers, therefore increasing the Seebeck coefficient. ${ }^{9}$ In other words, such barriers might induce an energy filtering effect, resulting in an increase in the Seebeck coefficient values. 


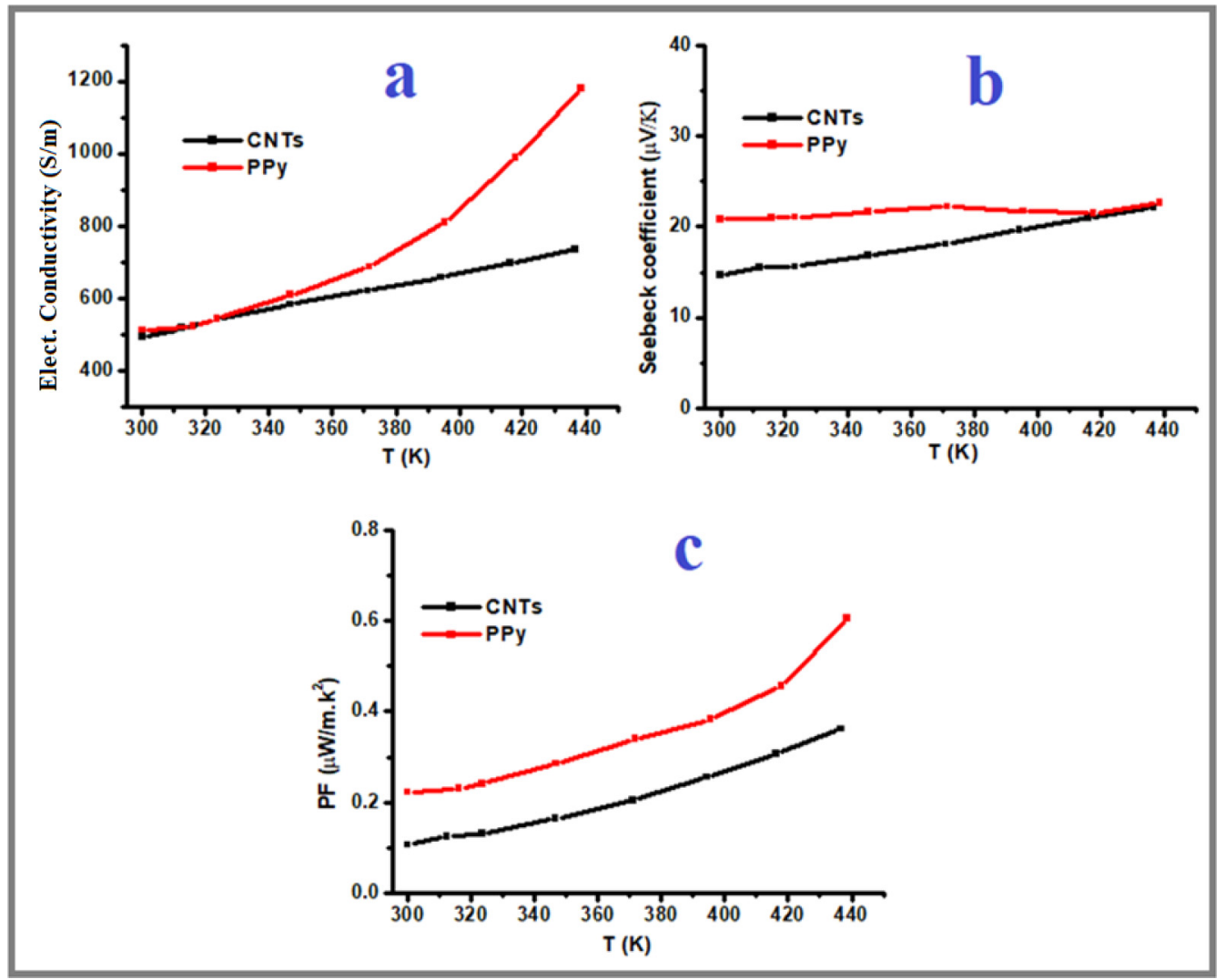

FIG. 9. TE properties of the CNTs and PPy.

The power factor $(P F)$ of both the CNTs and PPy as a function of temperature is shown in Fig. 9(c). At RT, the $P F$ values of the CNTs and PPy were 0.10 and $0.22 \mu \mathrm{W} / \mathrm{m} \mathrm{K}^{2}$, respectively. These values increased to 0.35 and $0.6 \mu \mathrm{W} / \mathrm{m} \mathrm{K}^{2}$, respectively, with an increase in temperature to $440 \mathrm{~K}$. The $P F$ value of the CNTs prepared in this study was relatively low as compared to those of the CNTs reported in the literature. ${ }^{12}$ This can be attributed to the low electrical conductivity of the CNTs prepared in this study. However, the $P F$ value of the PPy prepared in this study was much higher than those reported previously. ${ }^{11,26}$ This can be attributed to the high Seebeck coefficient of PPy. As shown in Figs. 2(a) and 2(b), the morphology of PPy showed a continuous sheet composed of tightly connected spherical nanoparticles. At the interfaces of the nanoparticles, potential barriers were formed, which allowed charge transport only for high-energy carriers. ${ }^{9}$ This induced an energy filtering effect, resulting in an increase in the Seebeck coefficient values.

The electrical conductivity, Seebeck coefficient, and power factor values of the CNTs with different PPy layer thicknesses are shown in Figs. 10(a)-10(c). The measured electrical conductivity (as a function of temperature) of the coated CNTs increased with an increase in the thickness of the coating layer. At RT, the electrical conductivity of the CNTs increased from approximately
$440 \mathrm{~S} / \mathrm{m}$ at the PPy amount of $0.05 \mathrm{ml}$ to approximately $1400 \mathrm{~S} / \mathrm{m}$ at the PPy amount of $0.3 \mathrm{ml}$ [Fig. 10(a)]. No further increase was observed in the electrical conductivity of the CNTs coated with $0.4 \mathrm{ml}$ PPy. The electrical conductivity of the coated CNTs showed a semiconductor behavior, e.g., an increasing trend with an increase in temperature. At $440 \mathrm{~K}$, the electrical conductivity values of all the coated samples were $25 \%-30 \%$ higher than those recorded at RT. This enhancement in the electrical conductivity of the CNTs by PPy coating is remarkable. This can be attributed to the smooth and perfect PPy coating formed on the surface of the CNTs. This coating facilitated charge transport and increased the charge concentrations simply by combining the carriers present in both the CNTs and PPy. These observations are consistent with those reported for PPy nanowire/graphene composites. ${ }^{26}$ The increase in the electrical conductivity of MWCNTs/PPy composites has also been reported in the literature ${ }^{11}$ and is attributed to the fact that MWCNTs can act as a template to guide the self-assembly of PPy into more ordered crystalline alignments via the $\pi-\pi$ interaction between PPy and the MWCNTs during processing. In this study, the CNTs were coated with PPy at different thicknesses. A systematic increase in the electrical conductivity was observed at the thinner layers, while the last thick layer of PPy on the CNTs $(0.4 \mathrm{ml} \mathrm{PPy})$ did not increase the conductivity further. It is possible 


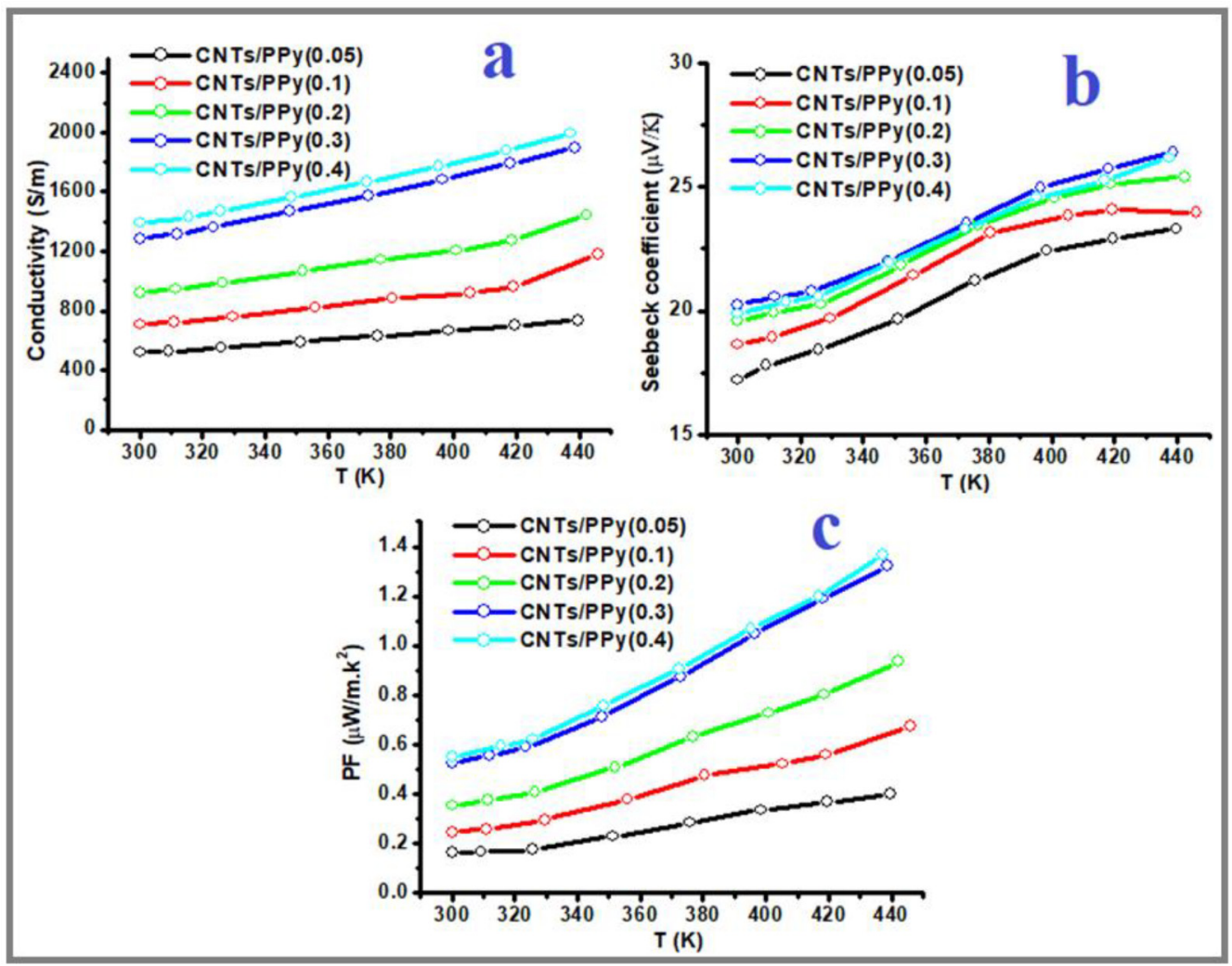

FIG. 10. TE performance of the CNTs with different PPy layer thicknesses.

that the concentration of the available carriers might be high at this layer, which could affect their mobility, thus reducing the electrical conductivity. The other most possible reason is the effect of energy filtering. This effect might be pronounced by the thick $0.4 \mathrm{ml} \mathrm{PPy}$ layer, which stopped any further increase in the electrical conductivity. The energy filtering effect perhaps was enhanced due to the considerable aggregates of PPy nanoparticles on the surface of CNTs, as shown in Fig. 3(f). These aggregates might further form energy barriers that scatter the low-energy charge carriers in the composite and allows only high-energy charge carriers to move across the interface.

The measured Seebeck coefficient and $P F$ values of the CNTs coated with PPy at different layer thicknesses shown in Figs. 10(b) and 10(c) showed a trend similar to that shown by electrical conductivity. It increased with an increase in the thickness of the coated layers. At RT, it increased from approximately $17 \mu \mathrm{V} / \mathrm{K}$ for the PPy amount of $0.05 \mathrm{ml}$ to approximately $21 \mu \mathrm{V} / \mathrm{K}$ for the PPy amount of $0.3 \mathrm{ml}$ [Fig. 10(a)]. No further increase was observed in the sample with $0.4 \mathrm{ml} \mathrm{PPy} \mathrm{coating.} \mathrm{At} 440 \mathrm{~K}$, the Seebeck coefficient values for all the coated samples were higher by approximately $25 \%$ than those recorded at RT. This increase was fast in the lower temperature region, $300-380 \mathrm{~K}$, and slowed down at higher temperatures. The temperature dependence of the Seebeck coefficient of the coated samples was almost similar to that of the pure CNTs [Fig. 9(b)]. Similarly, the $P F$ values of the samples coated with 0.3 and $0.4 \mathrm{ml}$ PPy increased rapidly with an increase in temperature, particularly above $350 \mathrm{~K}$. The $P F$ value of these two samples was approximately $0.5 \mu \mathrm{W} / \mathrm{m} \mathrm{K}^{2}$ at $\mathrm{RT}$, while it reached $1.4 \mu \mathrm{W} / \mathrm{m} \mathrm{K}^{2}$ at $440 \mathrm{~K}$. Although the CNTs showed low electrical conductivity as compared to those reported in the literature, ${ }^{36}$ the $P F$ value of the $0.3 \mathrm{ml} \mathrm{PPy-coated} \mathrm{sample} \mathrm{was} \mathrm{comparable} \mathrm{to} \mathrm{that} \mathrm{of}$ MWCNT/PPy composites. ${ }^{11}$

Figure 11 shows the thermal conductivity, $\kappa(\mathrm{a})$, and figure of merit, $z T$ (b), for the pure CNTs, PPy, and PPy-coated CNTs as a function of temperature. The thermal conductivity of the pure CNTs was approximately $1 \mathrm{~W} / \mathrm{mK}$ at RT. This value slowly increased to approximately $2 \mathrm{~W} / \mathrm{m} \mathrm{K}$ at $440 \mathrm{~K}$. This increase by heating might be due to the thermal expansion of these complex CNT structures, particularly at higher temperatures, which would facilitate the phonon transport and thus increase the thermal conductivity. However, such a low value has not been reported for CNTs but considerably higher values are well documented. ${ }^{37}$ This can be attributed to the zigzag bamboo-like structure of the CNTs (Fig. 1), which might have generated extra scattering sites for the phonons. The pure PPy showed a thermal conductivity value $(0.14$ $\mathrm{W} / \mathrm{m} \mathrm{K}$ at RT) close to those reported previously. ${ }^{9}$ This value remained constant with an increase in temperature up to $440 \mathrm{~K}$. However, in the case of the coated CNTs, the thermal conductivity 

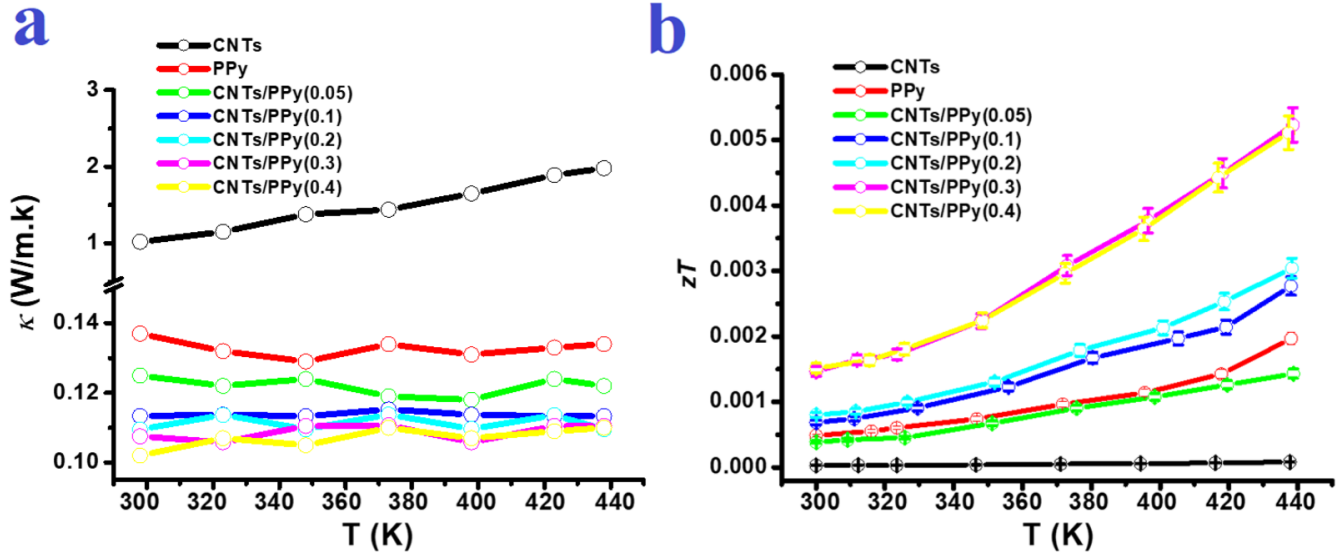

FIG. 11. Thermal conductivity, $\kappa(a)$, and figure of merit, $z T(b)$, of the CNTs with different PPy layer thicknesses as the functions of temperature.

value was further decreased by a factor of 10 for the CNTs coated with 0.3 and $0.4 \mathrm{PPy}$. This decrease can be attributed to the effect of the interfacial sites between the CNTs and PPy layer, which contributed to the scattering of more phonons. The formed aggregates of PPy in the surface of CNTs at higher amounts of PPy (e.g., 0.3 and $0.4 \mathrm{ml} \mathrm{PPy}$ ) might enhance the phonon scattering and reduce the thermal conductivity of the composite. It should be noted that the CNTs coated with PPy showed very low thermal conductivity as compared to similar composites reported in the literature.

The PPy coating significantly increased the figure of merit, $z T$, of the CNTs coated, as shown in Fig. 11(b). The $z T$ value of the pure CNTs was only $0.031 \times 10^{-3}$ at $\mathrm{RT}$, while it increased to $0.082 \times 10^{-3}$ at $440 \mathrm{~K}$. PPy showed higher $z T$ values $\left(0.5 \times 10^{-3}\right.$ at RT and $2.0 \times 10^{-3}$ at $\left.440 \mathrm{~K}\right)$ than the pure CNTs. The $z T$ values of the coated CNTs were significantly higher than those of both the pure CNTs and PPy. The highest $z T$ value of $1.5 \times 10^{-3}$ was obtained for the CNTs/PPy(0.3) and CNTs/PPy(0.4) samples at RT. This value increased to approximately $5.3 \times 10^{-3}$ at $440 \mathrm{~K}$. These preliminary results, which are still low as compared to those reported in the literature, demonstrate the potential of the low-cost oil fly ash-derived CNTs as TE materials.

The observed improvement in the TE performance of the PPy-coated CNTs caused by the significant increase in the electrical conductivity and Seebeck coefficient and by the reduction in the thermal conductivity can be attributed to several factors. These factors might include the large surface area of the CNTs, which served as conducting bridges or conducting networks connecting the PPy conducting domains. ${ }^{38}$ This increased effective percolation. ${ }^{39}$ The second factor might be related to the interaction between PPy and the CNTs. The third factor is that the CNTs act as a template to direct the self-assembly of PPy into more ordered crystalline alignments ${ }^{40}$ during the coating process, which improved the electrical conductivity of the CNTs. The fourth factor is related to the small improvement in the Seebeck coefficient values, which can be attributed to the energy filtering effect at the CNT/PPy interfaces, where appropriate potential boundary barriers favorably allowed the carriers with higher energy to pass, thereby increasing the mean carrier energy in the flow. ${ }^{11}$ One of the main findings of this study is the very low thermal conductivity of the PPy-coated CNTs. The complex network and the interfacial sites between the CNTs and the PPy layer might have a significant effect on scattering the phonons. All these factors might have contributed to improving the final TE performance of the PPy-coated CNTs.

It is reported that "polymer/carbon nanotube composites have lower electrical conductivity than pristine CNTs since the carrier barriers at the interface between the polymer and CNTs hinder the carrier pathways from tube to tube." ${ }^{41}$ In sharp contrast, the present work on coating CNTs of oil fly ash with PPy showed wellenhanced electrical conductivity. As mentioned above, this could be achieved due to the good interaction between PPy and CNTs through $\pi-\pi$ stacking between the polymer chains and these nanotubes. This could lead to a significantly strong coupling and easy charge transfer between the PPy and CNTs. The imperative approach adopted recently by Wang et $a l^{41}$ on forming ternary composites of polyaniline/polypyrrole/carbon nanotube and by treatment with a secondary dopant is attractive, as they significantly enhanced the electrical conductivity of this composite. However, the use of such complexes, which include long process and expensive products such as single-walled carbon nanotubes might be questionable. Moreover, the TE materials should possess low thermal conductivity, which has not been reported by Wang and his group. ${ }^{41}$ This part is very essential to understand the performance of a TE material, which should be demonstrated to calculate the $z \mathrm{~T}$ value of this doped ternary composite. On the other hand, the present work showed a good potential for CNTs of oil fly ash to be recommended as both a thermal insulator and TE material, particularly when coated with PPy.

A TE generator is a solid-state device employed to convert heat into electricity by the Seebeck effect. For oil fly ash-derived CNTs to be used in such devices, a number of factors must be considered. These include the output voltage, current, and power of the samples arising from the temperature gradient present between the two sides of a pressed compact. Therefore, the power generation 


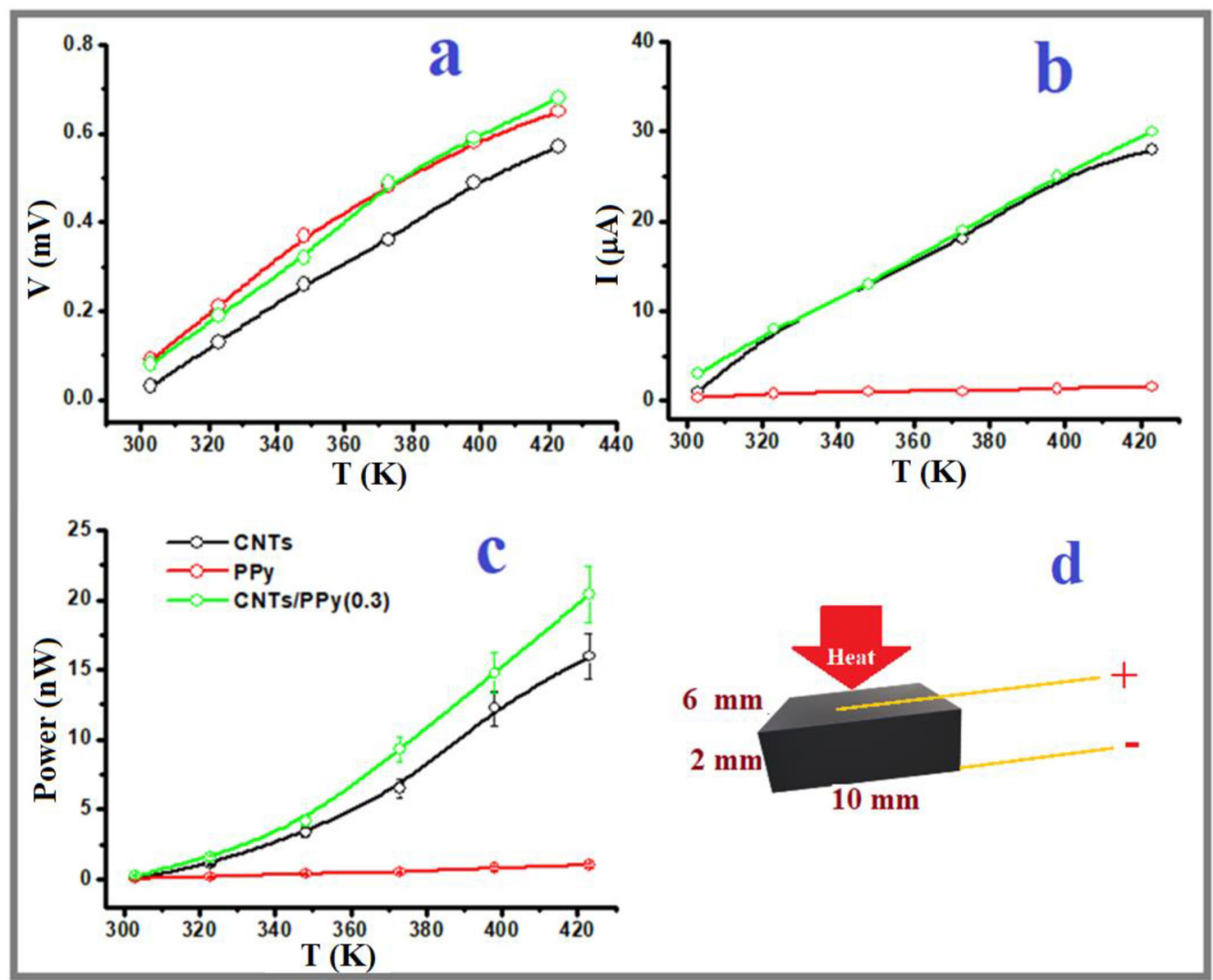

FIG. 12. Power generation characteristics of the single-leg modules made up of the CNTs, PPy, and CNTs/PPy(0.3) measured under real-time conditions in air. The measured output voltage (a), current (b), and power (c) of the modules as functions of the temperature. A single cubic leg module is shown in (d).

characteristics of the single cubic-shaped leg modules made up of the CNTs, PPy, and CNTs/PPy(0.3) were measured under real-time conditions in air, and the obtained results are shown in Fig. 12. The measured output voltage (a), current (b), and power (c) as functions of temperature showed that the PPy-coated CNTs showed improved power as compared to the individual samples. A single cubic leg module is shown in (d). The maximum voltage $(0.7 \mathrm{mV})$ and current $(30 \mu \mathrm{A})$ were achieved for the PPy-coated $\mathrm{CNTs}$ at $423 \mathrm{~K}$. At this temperature, the output power reached approximately $30 \mathrm{nW}(\Delta \mathrm{T}=30 \mathrm{~K})$. The obtained results were almost consistent with the $P F$ and $z T$ values of each leg. At higher temperatures (above $380 \mathrm{~K}$ ), the output power approximately 10 times higher than that at lower temperatures. Assessing the efficiency and stability of the power output of the PPy-coated CNTs under real-time conditions is essential for TE device applications.

The PPy coating not only enhanced the TE and power output of the CNTs but also acted as a good binder for the CNTs, which is necessary for making strong plates/legs. The results discussed here relate to only one leg of a p-type CNT coated with a PPy semiconductor. A useful electronic device with increased power could be designed using a large number of $\mathrm{p}-\mathrm{n}$ pairs with appropriate matching. The performance of oil fly ash-derived CNTs as a TE material can be improved by increasing the electrical conductivity by adding a small percentage of highly conducting materials or doping with proper activators, which will be considered in our future work.

\section{SUMMARY}

Oil fly ash-derived CNTs were successfully prepared and their TE performance was evaluated. The CNTs exhibited a unique structure and interesting TE properties. The TE properties of the CNTs improved with PPy coating. The uncoated CNTs exhibited a very low thermal conductivity of approximately $1 \mathrm{~W} / \mathrm{m} . \mathrm{K}$, which decreased drastically by a factor of 10 after the PPy coating. This result was validated by investigating the power generation characteristics of a single leg module made up of p-type PPy-coated CNTs. The results demonstrated the potential of the PPy-coated for application as TE materials. The low cost of these CNTs and their scalability is another attractive advantage, which might be utilized to design large TE plates as frames for house windows in hot countries. The low thermal conductivity of the PPy-coated CNTs 
might be quite useful for such plates to act as both heat insulators and TE power generators.

\section{ACKNOWLEDGMENTS}

This joint project was co-funded by the King Abdulaziz University (KAU), Jeddah, and King Abdullah University of Science and Technology (KAUST), Thuwal, under Grant No. JP-19-002. The authors, therefore, acknowledge KAU and KAUST for their technical and financial support.

\section{DATA AVAILABILITY}

The data that support the findings of this study are available from the corresponding author upon reasonable request.

\section{REFERENCES}

'T. Schulz, T. Reimann, A. Bochmann, A. Vogel, B. Capraro, B. Mieller, S. Teichert, and J. Töpfer, "Sintering behavior, microstructure and thermoelectric properties of calcium cobaltite thick films for transversal thermoelectric multilayer generators," J. Eur. Ceram. Soc. 38, 1600 (2018).

${ }^{2}$ R. R. Heikes and R. W. Ure, Thermoelectricity Science and Engineering (Interscience, New York, 1961).

${ }^{3}$ G. J. Snyder and E. S. Toberer, "Complex thermoelectric materials," Nat. Mater. 7, 105 (2008).

${ }^{4} \mathrm{~J}$. Cui, M. Wang, X. Xu, Y. Chen, and J. He, "Understanding the effects of iodine doping on the thermoelectric performance of $\mathrm{n}$-type $\mathrm{PbTe}$ ingot materials," J. Appl. Phys. 126, 025108 (2019).

${ }^{5}$ C.-J. Yao, H.-L. Zhang, and Q. Zhan, "Recent progress in thermoelectric materials based on conjugated polymers," Polymers 11, 107 (2019).

${ }^{6}$ M. S. Hossain, D. H. Huynh, P. D. Nguyen, L. Jiang, T. C. Nguyen, F. Al-Dirini, F. M. Hossain, and E. Skafidas, "Enhanced thermoelectric performance of graphene nanoribbon-based devices," J. Appl. Phys. 119, 125106 (2016).

${ }^{7}$ J. Kim, J. W. Roh, H. Moon, and W. Lee, "Observation of anisotropy in thermoelectric properties of individual single-crystalline bismuth nanowires," J. Appl. Phys. 122, 034303 (2017).

${ }^{\mathbf{8}}$ S. Iijima, "Helical microtubules of graphitic carbon," Nature 354, 56 (1991).

${ }^{9}$ L. Wang, F. Liu, C. Jin, T. Zhang, and Q. Yin, "Preparation of polypyrrole/ graphene nanosheets composites with enhanced thermoelectric properties," RSC Adv. 4, 46187 (2014).

${ }^{10} \mathrm{X}$. Wang, H. Wang, and B. Liu, "Carbon nanotube-based organic thermoelectric materials for energy harvesting," Polymers 10, 1196 (2018).

${ }^{11} \mathrm{~J}$. Wang, K. Cai, S. Shen, and J. Yin, "Preparation and thermoelectric properties of multi-walled carbon nanotubes/polypyrrole composites," Synth. Met. 195, 132 (2014).

12 J. L. Blackburn, A. J. Ferguson, C. Cho, and J. C. Grunlan, "Carbon nanotube-based thermoelectric materials and devices,” Adv. Mater. 30, 1704386 (2018).

${ }^{13}$ Y. Y. Tsai, J. S. Su, C. Y. Su, and W. H. He, "Production of carbon nanotubes by single-pulse discharge in air," J. Mater. Process. Technol. 209, 4413 (2009).

${ }^{14}$ A. Thess, R. Lee, P. Nikolaev, H. Dai, P. Petit, J. Robert, C. Xu, Y. H. Lee, S. G. Kim, A. G. Rinzler, D. T. Colbert, G. E. Scuseria, D. Tománek, J. E. Fischer, and R. E. Smalley, "Crystalline ropes of metallic carbon nanotubes," Science 273, 483 (1996).

${ }^{15}$ F. Kokai, I. Nozaki, T. Okada, A. Koshio, and T. Kuzumaki, "Efficient growth of multi walled carbon nanotubes by continuous-wave laser vaporization of graphite containing $\mathrm{B}_{4} \mathrm{C}$," Carbon 49, 1173 (2011).
${ }^{16}$ A. Vasylenko, J. Wynn, P. Medeiros, A. Morris, J. Sloan, and D. Quigley, "Encapsulated nanowires: Boosting electronic transport in carbon nanotubes," Phys. Rev. B 95, 121408 (2017).

${ }^{17} \mathrm{Z}$. Han and A. Fina, "Thermal conductivity of carbon nanotubes and their polymer nanocomposites: A review," Prog. Polym. Sci. 36, 914 (2011).

${ }^{18}$ N. A. Salah, "Method of forming carbon nanotubes from carbon-rich fly ash," U.S. patent 8,609,189 B2 (Dec. 17, 2013).

${ }^{19}$ N. Salah, S. S. Habib, Z. H. Khan, A. A. Al-Ghamdi, and A. Memic, "Formation of carbon nanotubes from carbon rich fly ash: Growth parameters and mechanism," Mater. Manuf. Processes 31, 146 (2016).

${ }^{\mathbf{2 0}}$ N. Salah, M. S. Abdel-Wahab, Z. H. Khan, and S. S. Habib, "Lubricant additives based on carbon nanotubes produced from carbon rich fly ash," Tribol. Trans. 60, 166 (2017).

${ }^{21}$ N. Salah, A. Alshahrie, M. S. Abdel-wahab, N. D. Alharbi, and Z. H. Khan, "Carbon nanotubes of oil fly ash integrated with ultrathin $\mathrm{CuO}$ nanosheets as effective lubricant additives," Diam. Relat. Mater. 78, 97 (2017).

${ }^{22}$ N. Salah, A. M. Alfawzan, A. Saeed, A. Alshahrie, and W. Allafi, "Effective reinforcements for thermoplastics based on carbon nanotubes of oil fly ash," Sci. Rep. 9, 20288 (2019).

${ }^{23}$ A. B. Zhang, B. L. Wang, J. Wang, J. K. Du, and C. Xie, "Effect of cracking on the thermoelectric conversion efficiency of thermoelectric materials," J. Appl. Phys. 121, 045105 (2017).

${ }^{24}$ L. Liang, G. Chen, and C.-Y. Guo, "Polypyrrole nanostructures and their thermoelectric performance," Mater. Chem. Front. 1, 380 (2017).

${ }^{25} \mathrm{~K}$. W. Shah, S.-X. Wang, D. X. Y. Soo, and J. Xu, "One-dimensional nanostructure engineering of conducting polymers for thermoelectric applications," Appl. Sci. 9, 1422 (2019).

${ }^{26}$ Y. Du, H. Niu, J. Li, Y. Dou, S. Z. Shen, R. Jia, and J. Xu, "Morphologies tuning of polypyrrole and thermoelectric properties of polypyrrole nanowire/graphene composites," Polymers 10, 1143 (2018).

${ }^{27} \mathrm{M}$. Maruthapandi and A. Gedanken, "A short report on the polymerization of pyrrole and its copolymers by sonochemical synthesis of fluorescent carbon dots," Polymers 11, 1240 (2019).

${ }^{28}$ A. H. P. de Oliveira, M. L. F. Nascimento, and H. P. de Oliveira, "Carbon nanotube@ $\mathrm{MnO}_{2} @$ polypyrrole composites: Chemical synthesis, characterization and application in supercapacitors," Mater. Res. 19, 1080 (2016).

${ }^{29}$ X. Fan, Z. Yang, and N. He, "Hierarchical nanostructured polypyrrole/ graphene composites as supercapacitor electrode," RSC Adv. 5, 15096 (2015).

${ }^{30}$ M. Šetka, R. Calavia, L. Vojkůvka, E. Llobet, J. Drbohlavová, and S. Vallejos, "Raman and XPS studies of ammonia sensitive polypyrrole nanorods and nanoparticles," Sci. Rep. 9, 8465 (2019).

${ }^{31}$ B. Zhang, Y. Xu, Y. Zheng, L. Dai, M. Zhang, J. Yang, Y. Chen, X. Chen, and J. Zhou, "A facile synthesis of polypyrrole/carbon nanotube composites with ultrathin, uniform and thickness-tunable polypyrrole shells," Nanoscale Res. Lett. 6, 431 (2011).

${ }^{32}$ M. A. Chougule, S. G. Pawar, P. R. Godse, R. N. Mulik, S. Sen, and V. B. Patil, "Synthesis and characterization of polypyrrole (PPy) thin films," Soft Nanosci. Lett. 01, 6-10 (2011).

${ }^{33}$ S. Rafique, R. Sharif, I. Rashid, and S. Ghani, "Facile fabrication of novel silver polypyrrole-multiwall carbon nanotubes nanocomposite for replacement of platinum in dye-sensitized solar cell," AIP Adv. 6, 085018 (2016).

${ }^{34}$ R. Bagai, J. Christopher, and G. S. Kapur, "Evaluating industrial grade functionalized multiwalled carbon nanotubes by X-ray photoelectron spectroscopy," Fullerenes Nanotubes Carbon Nanostruct. 27, 240 (2019).

${ }^{35}$ J. W. Lee, F. Serna, J. Nickels, and C. E. Schmidt, "Carboxylic acidfunctionalized conductive polypyrrole as a bioactive platform for cell adhesion," Biomacromolecules 7, 1692 (2006).

${ }^{36}$ A. Vasylenko, J. Wynn, P. V. C. Medeiros, A. J. Morris, J. Sloan, and D. Quigley, "Encapsulated nanowires: Boosting electronic transport in carbon nanotubes," Phys. Rev. B 95, 121408 (2017). 
${ }^{37}$ L. Zhu and B. Li, "Low thermal conductivity in ultrathin carbon nanotube," Sci. Rep. 4, 4917 (2014).

${ }^{38}$ M. Rahaman, A. Aldalbahi, M. Almoiqli, and S. Alzahly, "Chemical and electrochemical synthesis of polypyrrole using carrageenan as a dopant: Polypyrrole/ multi-walled carbon nanotube nanocomposites," Polymers 10, 632 (2018),

${ }^{39} \mathrm{~N}$. G. Sahoo, Y. C. Jung, H. H. So, and J. W. Cho, "Polypyrrole coated carbon nanotubes: Synthesis, characterization, and enhanced electrical properties," Synth. Met. 157, 374 (2007).
${ }^{40}$ J. Luo, S. S. Jiang, Y. Wu, M. L. Chen, and X. Y. Liu, "Synthesis of stable aqueous dispersion of graphene/polyaniline composite mediated by polystyrene sulfonic acid," J. Polym. Sci. A Polym. Chem. 50, 4888 (2012).

${ }^{41}$ S. Wang, Y. Zhou, Y. Liu, L. Wang, and C. Gao, "Enhanced thermoelectric properties of polyaniline/polypyrrole/carbon nanotube ternary composites by treatment with a secondary dopant using ferric chloride," J. Mater. Chem. C 8, $528(2020)$. 